Article

\title{
Tracking and Monitoring System Based on LoRa Technology for Lightweight Boats
}

\author{
Ramon Sanchez-Iborra ${ }^{1, * \mathbb{D}}$, Ignacio G. Liaño ${ }^{2}$, Christian Simoes ${ }^{2}$ and Elena Couñago ${ }^{2}$ \\ and Antonio F. Skarmeta ${ }^{1}$ \\ 1 Department of Information and Communications Engineering, University of Murcia, 30100 Murcia, Spain; \\ skarmeta@um.es \\ 2 Centro Tecnológico del Mar-Fundación CETMAR, 36208 Vigo, Spain; igonzalez@cetmar.org (I.G.L.); \\ csimoes@cetmar.org (C.S.); ecounago@cetmar.org (E.C.) \\ * Correspondence: ramonsanchez@um.es; Tel.: +34-868-884-644
}

Received: 15 November 2018; Accepted: 20 December 2018; Published: 22 December 2018

\begin{abstract}
Maritime communications are really challenging due to the adverse transmission conditions and the lack of a pre-provided infrastructure for supporting long range connectivity with land. Communications in high seas are usually covered by satellite links that are expensive and lead to high power consumption by the terminals. However, in areas closer to the shore, other communication options have been adopted for different kinds of services such as boat tracking and telemetry, data collection from moored monitoring systems, etc. In these scenarios, technologies such as cellular communications or wireless sensor networks have been employed so far; nevertheless, all of them present different drawbacks mostly related with the coverage and energy-efficiency of the system. Recently, a novel communication paradigm, so-called Low Power-Wide Area Network (LP-WAN) has gained momentum due to its interesting characteristics regarding transmission distances and end-node's power consumption. The latter may be of great interest for ships with energetic restrictions such as small sailboats, recreational boats, or radio control ships. For that reason, in this work, we present a boat tracking and monitoring system based on LoRa (Long Range), one of the most prominent LP-WAN technologies. We provide a comprehensive overview of this communication solution as well as a discussion addressing its benefits when applied to maritime scenarios. We present the results extracted from a case of study, where real-training sessions of Optimist Class sailboats have been monitored by means of the presented architecture, obtaining good levels of coverage and link-reliability with limited power consumption. A transmission range study is also presented, demonstrating the validity of this proposal for monitoring activities inside the port or maneuvers close to the shore.
\end{abstract}

Keywords: maritime communications; intelligent transportation systems (ITS); LoRa; low power-wide area network (LP-WAN); internet of things (IoT); energy efficiency

\section{Introduction}

Enabling maritime communications have been a big challenge since their origins [1]. Although radio transmission technologies have rapidly evolved during the last times, e.g., WiFi, 4G, WiMAX, etc., maritime scenarios still present particular characteristics that notably make more difficult the deployment of reliable and widely adopted communication systems. There are many services and operations that depend on a trustworthy communication architecture, e.g., ship/boat monitoring, maintenance of navigation marks, goods tracking, data extraction from sensing platforms, unmanned vehicle management, safety operations, voice communications, etc. [2]. 
Depending on their distance to the shore, two well-differenced scenarios have been identified within the maritime communications ecosystem. Thus, sea areas tens of miles apart from land are considered long range scenarios, while closer areas are considered medium or short range scenarios [3]. Regarding the former, few communication options are available for providing these areas with coverage. Basically, they are based on satellite communications [4], e.g., Argos, Iridium, or Iridium NEXT, or the classic Very/Ultra High Frequency (VHF/UHF) radio links [5]. These technologies provide ships with basic and low data-rate communication capabilities, which in many cases demand a high expenditure. Nevertheless, the focus of this work is on the second case, namely, communications in coastal areas. For these scenarios, a larger set of solutions have been employed for covering them with wireless connectivity. Depending on the application requirements or the boat characteristics, different technologies have been adopted. In the case of boats or ships without payload or power consumption restrictions, cellular communications or WiMAX technologies have been proposed [1,6]. However, other scenarios such as those comprising sailboats, small autonomous vehicles, or moored sensing platforms, among many others, present severe energetic restrictions as the communication modules are usually powered by batteries. Note that the transmission technologies cited above are not battery friendly as they were not designed for transporting low data-rate/bandwidth communications like those typical in Internet of Things (IoT) scenarios [7]. For that reason, alternative communication technologies are needed for providing these restricted nodes with connectivity with land. Examples of these technologies are those based on Wireless Sensor Networks (WSN) [8], which are battery friendly but present other drawbacks related to their short range that increase the WSN management complexity, e.g., multi-hop routing [9].

A novel communication technology that is attracting the attention of both academia and industry is the so-called Low Power-Wide Area Network (LP-WAN). This solution is highly appreciated for IoT applications because it enables long transmissions of more than $15 \mathrm{~km}$ in free space [10] with limited power consumption that permit end-node battery's lifetimes of years [7]. Figure 1 shows a comparison of the transmission range and energy efficiency of the communication technologies discussed above, including LP-WAN. Many maritime applications, as some of those previously cited, perfectly match the main IoT systems precepts: (i) need for long range transmissions; (ii) reduced use of bandwidth per message; (iii) limited number of messages per node per day; (iv) big number of simultaneously connected end-devices, and (v) low-cost end-devices. All these characteristics are satisfied by the wide plethora of LP-WAN-based platforms; however, among all of them, LoRa (Long Range) [11] is one of the most prominent so it is receiving great attention by the research community $[10,12]$.

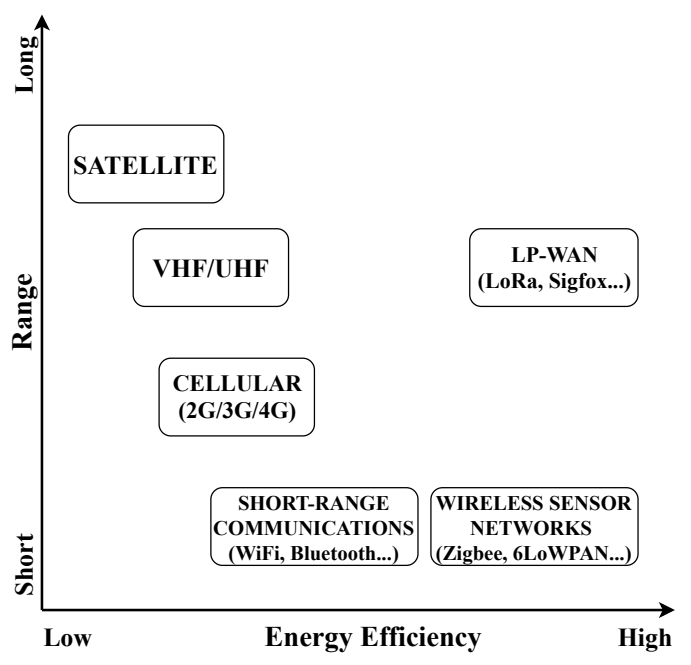

Figure 1. Comparison of transmission range and energy efficiency provided by different communication technologies employed in maritime scenarios. 
Therefore, in this work, we present LoRa as a promising enabler for certain maritime activities performed in coastal areas. As stated above, monitoring or tracking activities and safety messaging are applications that may be simply enabled by using this type of transmission technology. In addition, we present a real deployment with the aim of monitoring Optimist Class sailing races. The Optimist is an official international class [13] that embraces small sailboats with hull length and weight of $2.3 \mathrm{~m}$ and $35 \mathrm{~kg}$, respectively. The Optimist is the biggest youth racing class in the world with an annual world championship. Thus, the proposed system has been tested by monitoring a series of training sessions of Optimist Class sailboats by means of LoRa-enabled communication between the boats and a base station placed in the port. The developed monitoring platform permits tracking not just each boat position but also key environmental and boat parameters. Thus, the main contributions of this work are the following: (i) a deep discussion of LP-WAN and its applicability to maritime scenarios; (ii) an experimental equipment for monitoring both oceanic and boat parameters; and (iii) the validation of a real deployment by which the monitoring of sailboat activities through the Internet is enabled.

The rest of the paper is organized as follows. Section 2 reviews relevant work regarding the use of IoT-based communication technologies in maritime scenarios. The main features of LoRa technology are deeply explored in Section 3. Section 4 discusses the main drawbacks of IoT-based communication technologies when applied to coastal scenarios and presents the potential benefits of employing LP-WAN solutions for maritime communications. Section 5 details the test-bench employed for our real deployment. Section 6 presents and discusses the attained results extracted from our validation test. Finally, the paper ends by summarizing the most important findings in Section 7.

\section{Related Work}

As stated previously, different communication alternatives usually employed in IoT deployments have been applied to maritime scenarios. These technologies include WSN, such as IEEE 802.15.4-based architectures, cellular systems, e.g., GSM (Global System for Mobile communications), 3G, and 4G, or VHF/UHF-based solutions [2]. However, very few works have addressed the use of LP-WAN technologies in these scenarios. In the following, an overview of different recent efforts performed for establishing maritime communications with these technologies is presented.

Regarding WSN, they have been used for many applications due to their energy efficiency that permits end-nodes to be alive during years without battery replacement. Thus, works in $[14,15]$ proposed employing Zigbee-based WSNs for maritime surveillance and safety monitoring. However, both papers adopted different approaches. The first one [14] presented a simulation-based framework for developing a maritime sensor network. With the aim of implementing a system for acquiring information about ship dynamic states, marine environmental protection, etc., the authors introduced the needed architecture and communication protocols based on WSN. In turn, the work in [15] showed the real deployment of a long-term hazard monitoring system installed in a harbor. The nodes composing the WSN made use of an energy-saving smart algorithm and data encryption for securing the Zigbee link between them and the gateway. Although less energy efficient, WiFi technology has been proposed too in other real deployments $[16,17]$.

In turn, Mobile Ad hoc NETworks (MANET) have also been explored in maritime scenarios $[18,19]$. Work in [18] proposed a Nautical Ad hoc NETwork (NANET) in the VHF band in order to improve the range of the individual links. Depending on the distance of the nodes to the shore, the authors proposed to employ terrestrial nodes for enabling the communications in coastal areas and the use of P2P (ad hoc) links in further areas to the shore. From a different perspective, the authors of [19] studied the impact of typical maritime traffic patterns on the performance of different MANET routing protocols. The results showed that, in some scenarios, a boat chain can be established for transporting information from a far node to the gateway on land in a multi-hop fashion, but, in other cases, some boats are isolated with no communication capabilities. In order to solve the issue of temporally isolated nodes due to frequent disconnections, the use of Delay Tolerant Networks (DTN) have been proposed as well $[20,21]$. Work in [20] explored the use of WiMAX-based technology for over the horizon 
ship-to-ship communications following a DTN scheme. The results, attained by means of computing simulation, showed superior performance of DTN-oriented routing protocols in comparison with traditional MANET routing algorithms. In turn, the authors of [21] also made use of simulation tools to demonstrate that DTN are a viable option, but, even more important, that as vessel mobility patterns can be well-known beforehand, the upcoming network topology changes can be accurately predicted. Considering this fact, more efficient routing algorithms can be developed within the context of DTN.

As discussed later, cellular networks are not the best option for constrained devices because of the associated high power consumption of the communication module; despite this, they have been employed in maritime scenarios due to their longer range in comparison with WSN technologies. As a typical usage example, the authors of [22] used GSM and 3G links to connect a set of Autonomous Underwater Vehicles (AUV) and wavy drifter buoys with monitoring purposes to the data collector. Other works [2] have identified cellular technologies to be more appropriate in the case of large boats or ships because they do not present energetic restrictions and may employ high-gain antennas in order to extend the link with the base station on land.

Finally, as stated previously, some recent works have employed LP-WAN technologies in maritime scenarios [23-25]. The work in [23] proposed a distributed sensors system with the aim of controlling moorings in marinas. Due to the characteristics of the adopted communication technologies, the authors could deploy a system with a relatively large number of nodes simultaneously communicating with a central point. The authors of [24] presented a short paper in which a LoRa-based sailing monitoring system was implemented. In this study, just one LoRa configuration was evaluated, namely, the one with the greatest data-rate. As discussed in Section 3, employing high bit-rates in LP-WAN systems leads to decreasing the radio-link robustness; therefore, a limited transmission range of $2 \mathrm{~km}$ was attained in this experiment. In the present work, we have also evaluated another LoRa configuration that provides greater reliability to the wireless transmission, permitting longer transmission ranges as shown later. Finally, the authors of [25] showed good performance of a LoRa transmission system in an open-sea environment by evaluating the transmission range of an end-device on-board a ship with respect to a gateway located in land. Different from this work, we present an end-to-end deployment (from the boat to the remote user) aiming at monitoring sailing activities close to the shore or without leaving the port. Concretely, in our validation test, the communication modules have been placed on-board a number of sailboats with heavy payload and energetic restrictions which may be the main target of application for LP-WAN systems in maritime scenarios. In this line, we have taken into consideration the power consumption of end-devices too. Using this deployment, we have monitored a series of Optimist Class training session with the aim of enabling the visualization through the Internet of a real race with a high number of nodes sending data about their status and location as well as environmental metrics.

In the following sections, we explore the different components of this deployment and the benefits of employing an LP-WAN solution in this case of study and other applications.

\section{LoRa}

LoRa is a novel transmission technology that enables long communications of more than 10 miles with reduced power consumption that permits battery's lifetimes of about 10 years [7]. To achieve these goals, LoRa makes use of a series of strategies: (i) reduced transmission bit-rates; (ii) use of low frequency bands (sub-GHz bands); and (iii) limited end-devices' communication capabilities. Regarding the former, LoRa employs limited data-rates of few kilobits per second (kbps) that allow the use of high-sensitive receivers. Thereby, the receiving equipment is capable of decoding the transmitted information when it is received at a very low signal-level. This transmission robustness is enabled by the characteristics of the LoRa modulation, which is a proprietary modulation by Semtech (Camarillo, CA, USA) based on the Chirp Spread Spectrum (CSS) modulation technique [26]. The use of low frequency bands ( $868 \mathrm{MHz}$ and $911 \mathrm{MHz}$ in Europe and America, respectively) improves the transmission range and penetration in comparison with other typical higher frequency bands such 
as $2.4 \mathrm{GHz}$ [27]. In addition, low frequency instruments are cheaper than those operating at higher frequencies. It is important to note that LoRa uses Industrial, Scientific, and Medical (ISM) frequency bands, which are unlicensed, so anyone can freely transmit on them. Finally, in order to save energy, the communications in LoRa systems are limited in two different ways. First, the use of unlicensed frequency bands that host a great amount of devices, so that the number of messages that each node can send per day is limited for avoiding the overload of the band (duty cycle). In addition, the payload length of each packet is also limited due to the low data-rate of the transmissions. The use of long packets would lead to very long time-on-airs hence occupying the shared transmission medium for a very long time. Observe that both transmission limitations help to reduce the power consumption of the communication modules.

One of the most valued characteristics of LoRa is its adaptability to different transmission needs. To this end, LoRa presents three configuration parameters, namely, Spreading Factor (SF), Coding Rate (CR), and bandwidth (BW). The SF indicates the grade of spreading of the transmitted signal with respect to the original one-the longest spreading, the greatest transmission robustness, but the lowest effective data-rate. The CR determines the amount of redundant information included in the transmitted packet. This extra information is employed for reconstructing the received data in case of receiving the packet partially corrupted. Employing high CR leads to an improved system robustness but at the expense of introducing extra overhead in the communication process. Finally, LoRa permits the use of different BW, although the most employed is $125 \mathrm{kHz}$. Other values such as $250 \mathrm{kHz}$ or $500 \mathrm{kHz}$ are also supported.

From a network architecture perspective, there is an upper layer so-called LoRaWAN (LoRa Wide Area Network) that defines the network topology or security mechanisms, among other aspects (Figure 2). Similar to all LP-WAN solutions, LoRaWAN proposes a star or star-of-stars topology (Figure 3). This architecture is enabled by the long links established between the gateway or base station and the end-nodes. This configuration avoids complex network organizations such as mesh or hierarchical networks because the end-devices directly communicate with the base station without the support of the rest of nodes composing the network. Note that, although in our real deployment we adopted the LoRaWAN architecture (all the boats directly connected to a common gateway), from a networking point of view, we just made use of the LoRa layer, hence avoiding extra overhead introduced by LoRaWAN.

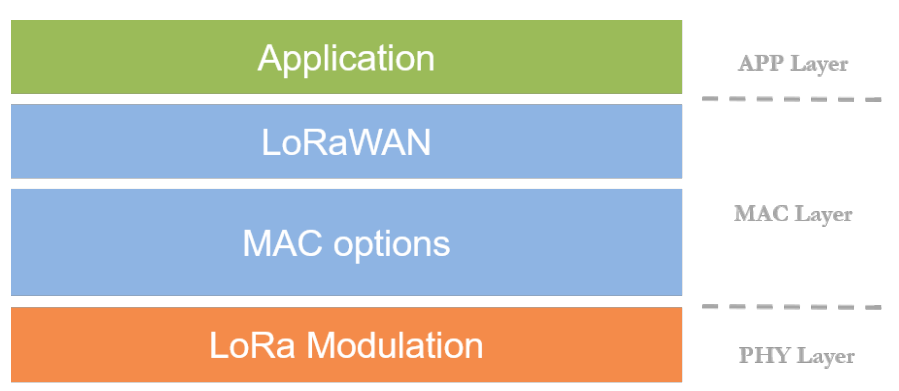

Figure 2. LoRaWAN (Long Range Wide Area Network) stack. 


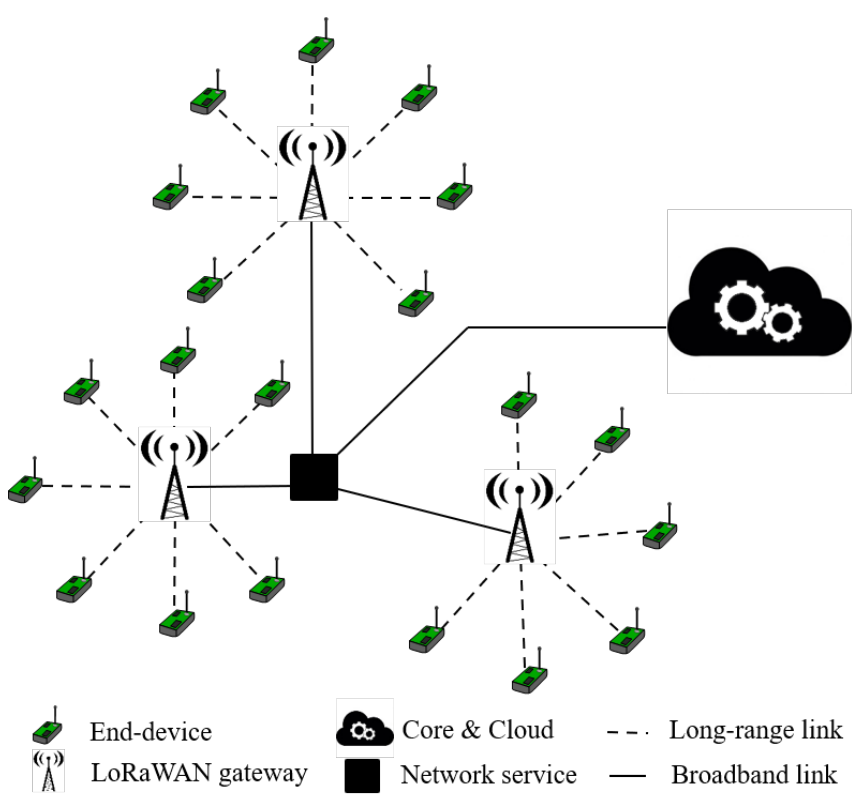

Figure 3. LoRaWAN network architecture.

\section{IoT-Based Communication Technologies in Coastal Scenarios}

In the following, firstly, the main restrictions of current IoT-based communication technologies employed in maritime scenarios are identified; thereafter, potential applications that may take advantage of using an LP-WAN communication system are explored. Finally, an LP-WAN-based architecture is proposed.

\subsection{Limitations on Existing Solutions}

As mentioned above, so far, two different families of IoT-based communication technologies for sensing and monitoring applications in coastal scenarios have been employed, namely, short and medium range technologies. However, these communication technologies present notable impediments for deploying sustainable and cost-effective solutions: (i) transmission range, which notably determines the network organization and its scalability, (ii) edge-nodes' dimensioning and power efficiency, and (iii) network management costs [7]. Please note that we do not consider satellite communications as an enabling technology in these scenarios due to its high cost and energy consumption, which clearly differ from the basic requirements of IoT systems.

Regarding short range technologies, WSN solutions such as ZigBee or 6LoWPAN have been adopted in many scenarios $[14,15]$. The main drawback of these alternatives is their restricted transmission range, which severely limits the operation area and increments the complexity of network management tasks. The use of this kind of solutions implies the configuration of a multi-hop network in order to send the information to a collection point or using other alternatives even more complex and expensive. In turn, medium range communication technologies such as cellular systems, e.g., GSM or 3G, have also been employed for providing ships or monitoring platforms with connectivity [22]. However, although this approach permits longer radio-links, it presents important deficiencies in order to be directly used by constrained end-devices. They are motivated by the initial design of cellular technologies, which were focused on human-type communications, i.e., they provide broadband access to a limited number of users. Nevertheless, unlike this scheme, typical monitoring scenarios will host a notable number of devices generating short packets sporadicly. In addition, current cellular technologies are not battery friendly either, which is a critical requirement of unattended maritime deployments. 


\subsection{Application of LP-WAN Technologies}

In the light of the previous discussion, LP-WAN-based solutions, e.g., LoRa, rise as a promising alternative to enable long-term, scalable, and low-cost monitoring deployments for maritime environments. As discussed previously, the main limitations of these systems are their transmission restrictions in terms of packet length and data-rate. However, a number of coastal applications may fit well within these restrictions as the information to be transmitted and its periodicity might be low.

Monitoring and alerting operations for boats and ships in coastal areas are services that can be perfectly supported by LP-WAN technologies. Thus, boats may keep port authorities and safety services periodically informed about their state and position. Although, in some cases, energy restrictions will not be an issue, the communication range will be enlarged to distances not covered by any other kind of communication technology (except satellite ones). In addition, by using a device-to-device architecture, boats can communicate with each other in case of losing connectivity with land. In the special case of ships with energetic restrictions, which is the main focus of this work, LP-WAN radio modules may fit very well due to their limited power consumption.

Another potential field of application for LP-WANs is the monitoring of maritime of autonomous vehicles, e.g., Unmanned Surface Vehicles (USV), Unmanned Aerial Vehicles (UAV), or unmanned ships/ferries, which have been gaining great momentum in recent years [2]. Although the communication channel for managing and remote controlling operations demands low latency and certain transmission rate that are not attainable by LP-WAN solutions, this alternative communication link may be used for data-retrieving or tracking activities. These vehicles have been used as monitoring stations themselves or as collecting points for gathering data generated by elements without connectivity to land [22]. Thus, by using a P2P-based architecture of LP-WAN links [28], the transmission distance between both ends can be notably reduced, hence reducing the traveling time and distance.

Finally, moored platforms or buoys are also widely employed for monitoring both environmental conditions and oceanic metrics. Without a proper communication technology, these systems make marine researchers to manually collect the data sampled by the monitoring system. This strategy is also prone to suffer information loss or delay in the data collection due to adverse climatic conditions. For that reason, by installing a low-cost LP-WAN-based transmission module, this task can be automatically scheduled, hence improving the frequency and easiness of this process. Please observe that this monitoring platforms should have long life periods, which is also supported by the long battery life-times of the proposed communication system.

\subsection{Proposed Architecture}

Henceforth, an LP-WAN-based end-to-end architecture is proposed in order to (i) retrieve data from ships and (ii) present them through some kind of data visualization platform or control system. This architecture is shown in Figure 4. It has been divided in three different areas, namely, Radio Access Network (RAN), cloud, and user domain. As explained in the previous section, the RAN is enabled by the use of an LP-WAN solution that permits the communication between ships and the base station/gateway in land. This is the most challenging part of the architecture due to the adversity that may be introduced by propagation and scenario conditions. The gateway is the contact point that forwards the received data to the cloud. This intermediate section of the architecture includes a distribution network, e.g., the Internet, that delivers these data to a central server in charge of their aggregation. At this point, several services may be implemented from a parameters' visualizer to more complex applications based on data analytics or alert systems. In this part of the architecture is where the system intelligence resides. Finally, the processed data is presented as useful information to the users, enabling its visualization or even interaction depending on the service under consideration. This way, the end-users of this architecture are able to accurately monitor the maritime activities, including ships' positions and other sensed parameters. 


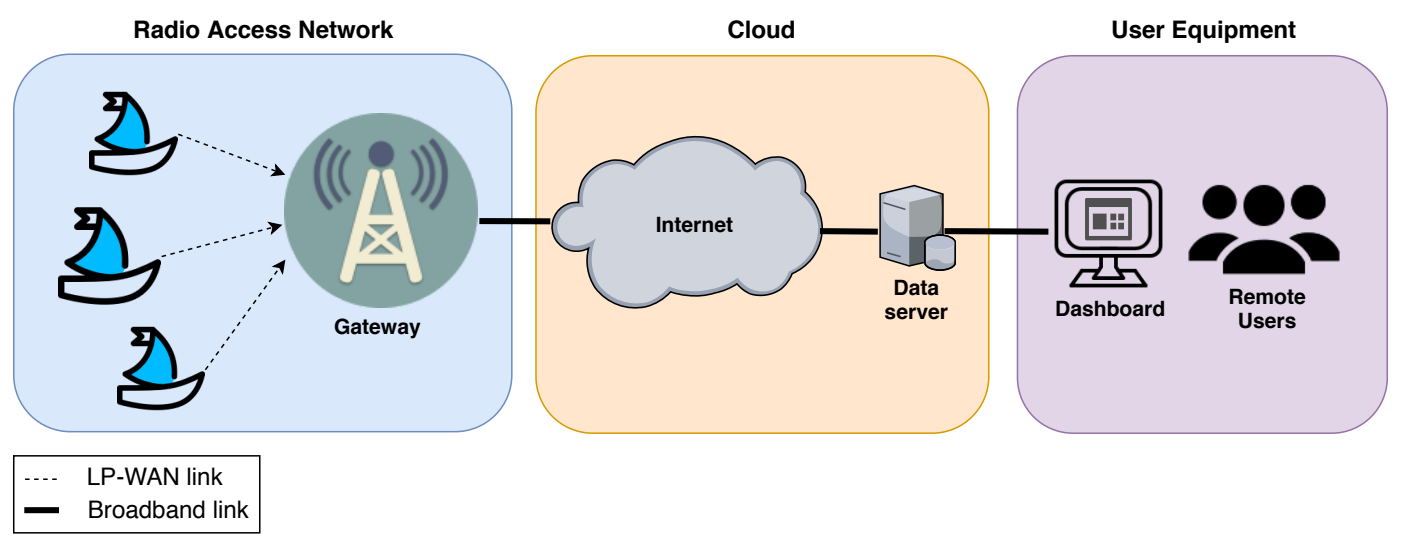

Figure 4. LP-WAN-based proposed architecture.

\section{Test-Bench}

In this section, we first present the scenario and context of our validation tests. Thereafter, the equipment employed for the experimental setup is comprehensively explored.

\subsection{Scenario}

As explained before, the designed and implemented system is devoted to monitor and report the evolution of races of light and small boats. In this case, we installed our LoRa-enabled monitoring devices on-board of Optimist Class sailboats (Figure 5) in order to track part of their training sessions in the port of Vigo (Spain) (Figure 6). Concretely, we monitored a series of training sessions of $1 \mathrm{~h}$ of duration with maximum transmission distances of $1 \mathrm{~km}$ inside the port. As will be detailed in the following section, the boats were equipped with a set of sensors and a LoRa transceiver with the aim of enabling the transmission of their position and other additional metrics to a base station placed on the roof of a building in the port (Figure 7). The nature and inherent limitations of these boats pose important energetic and payload restrictions, so both the equipment and the transmitted data were optimized to accomplish with these requirements. Due to the big attention that these races are receiving during the last times, a tracking platform through the Internet has also been developed. Thus, the base station uploads the received data to a service hosted in the cloud that presents the race metrics to the users by means of a web-based dashboard (Figure 8). Observe that this dashboard automatically updates the position and other metrics for each boat, so the race can be followed live with a certain delay added by the LoRa link. This delay appears because, as explained above, LP-WAN technologies offer low-data rates that introduce certain latency in the data transmission, although this delay is assumable for the application under consideration. This fact will be deeply discussed in Section 6. In the following, we examine the technical details of the mentioned deployment.

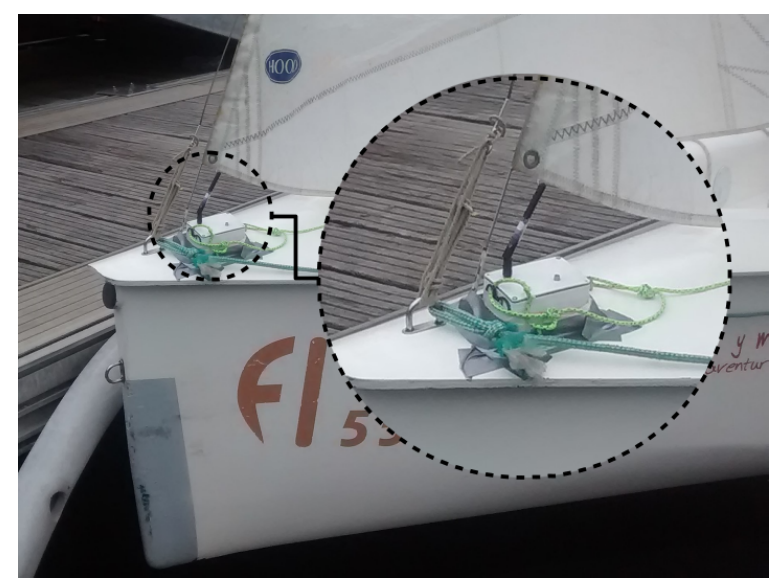

Figure 5. Tracking module on-board a participant boat. 


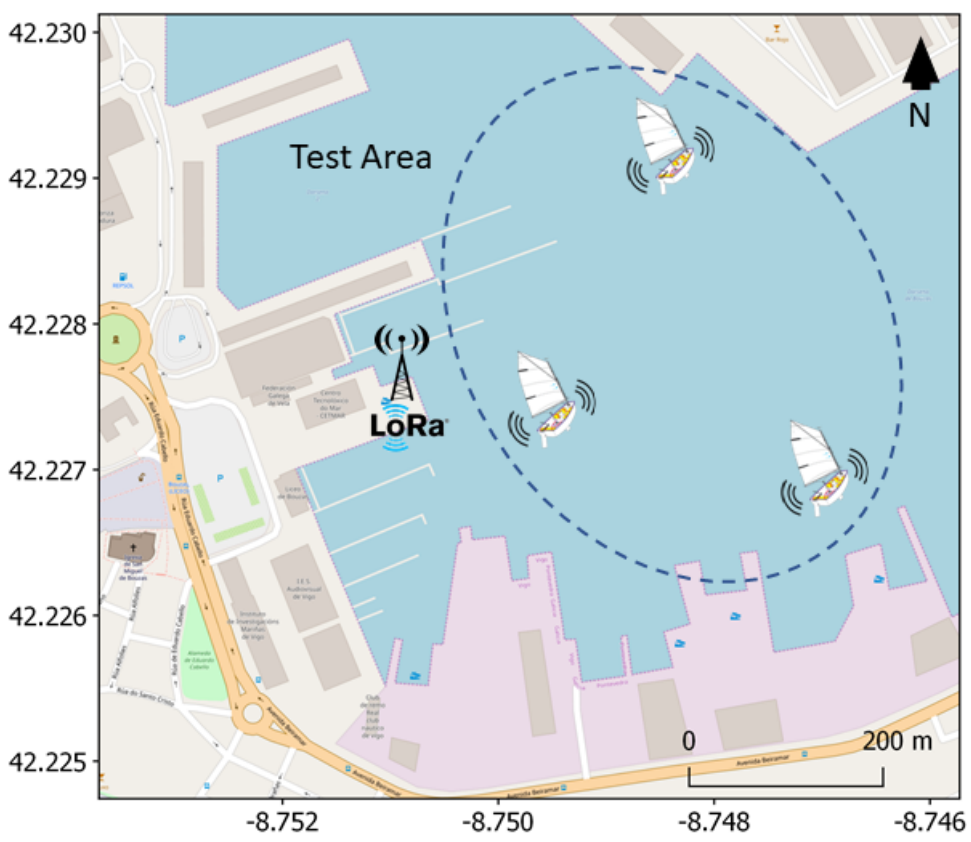

Figure 6. Test scenario in the port of Vigo (Spain).

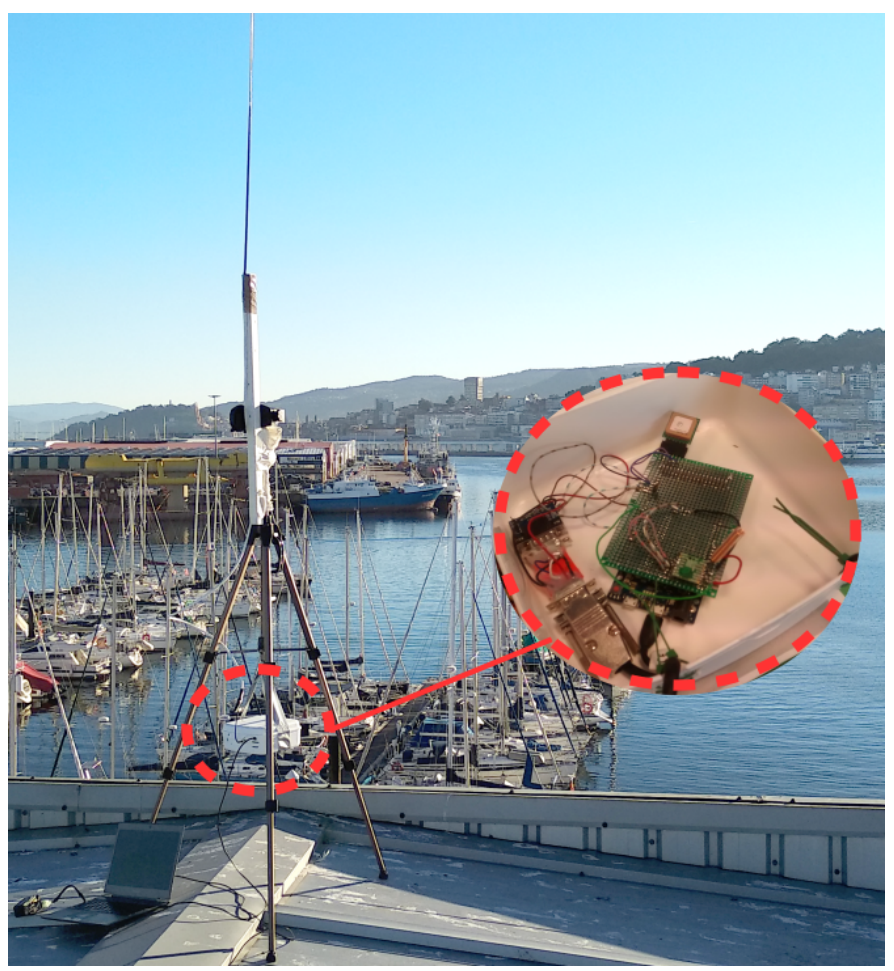

Figure 7. Base station. 


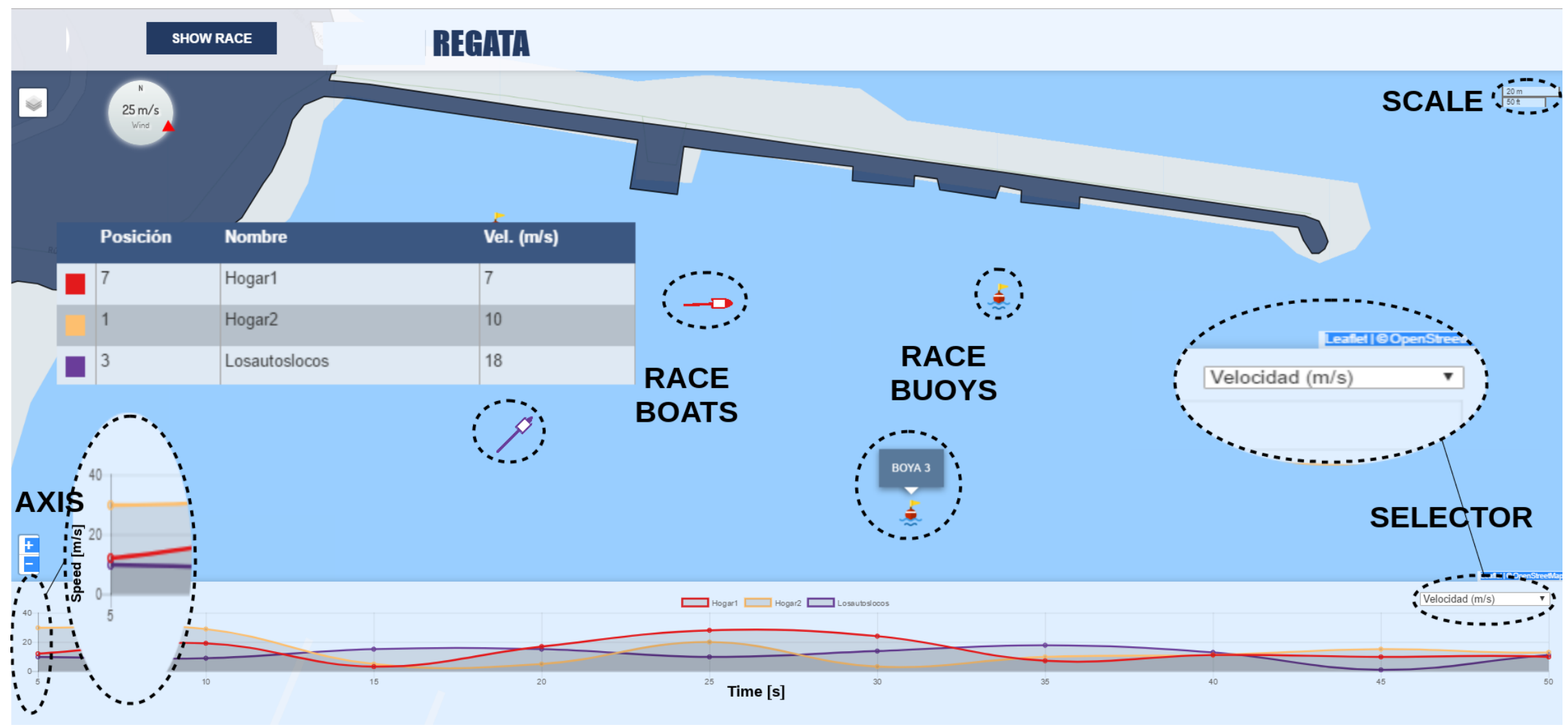

Figure 8. Dashboard for the online race monitoring. 


\subsection{Equipment}

As aforementioned, a LoRa-enabled monitoring unit was placed in the bow of the tracked boats (Figure 5). This module consisted of an Arduino mini Pro board operating at a frequency of $16 \mathrm{MHz}$, to which the rest of the sensors were connected, namely, a NeoN8M GPS module by U-BLOX (precision of $2 \mathrm{~m}$ ) (Thalwil, Switzerland), an MPU9250 inertial unit (InvenSense, San Jose, CA, USA), a BMP280 barometric sensor (Bosch Sensortec, Reutlingen/Kusterdingen, Germany), an ACS712 amperimetric sensor (Allegro MicroSystems, Worcester, MA, USA), and a Light Dependent Resistor (LDR) to measure the received light intensity. Regarding the LoRa-based communications, a SX1278 transceiver (Semtech (previously defined in page 4)) was used with an output power of $14 \mathrm{~dB}$ and a transmission channel in the $434 \mathrm{MHz}$ band. All of these devices were encapsulated in a case of $60 \times 70 \times 50 \mathrm{~mm}$ (see Figure 5). Attached to this case, the employed antenna was an $1 / 4$ wave helical dipole. It is important to note that, due to the structural restrictions of the boats under consideration, the installation of the device was always on the bow of the ship, which implied that the position of the antenna never exceeded 80 $\mathrm{cm}$ above the sea level. As explained in the next section, the water proximity to the communication equipment may introduce some additional adversity to the propagation conditions.

The communications with land were established through a base station placed in the port (Figure 7). This base station was deployed by means of an Arduino Due board with the same GPS module and LoRa device as those installed in the end-devices. A full wave antenna was attached to the SX1278 module. In this case, the antenna was not directly connected to the transceiver, but a wire of $1.5 \mathrm{~m}$ was employed. The base station was installed on the terrace of a building at the edge of the sea. With this configuration, it presented a height of about $3 \mathrm{~m}$ above the sea level. Please note that the there was no line of sight at every measurement point in the studied area, as discussed later.

From a software perspective, the base station implemented self developed routines written in Python, for packet decoding and its subsequent storage in a PostGis database. In addition, in order to present the results through the web-based dashboard (Figure 8), an own Javascript development was employed. Observe that, in this monitoring panel, race buoys and boat position and their rankings are shown at the top of viewer. The table includes the entire list of participants and the instantaneous velocity of each boat. On the top of this chart, wind speed and its direction are also presented. At the bottom, a plot shows the evolution over time of several eligible variables, e.g., speed, temperature, light intensity, or power consumption, among others. The messages generated by the monitoring units presented the structure showed in Figure 9 with a full length of 52 Bytes, which permitted us to transmit the information gathered by the on-board sensors to the base station. After a control header of 8 bytes, the payload field included the following data: instant GPS position, instant Integer Time Of Week (ITOW), instant heading and its accuracy, average and standard deviation of trajectory orientation, average and standard deviation of gyroscope $(x, y, z)$, average and standard deviation of accelerometer $(x, y, z)$, average and standard deviation of incident light, average and standard deviation of pressure, average and standard deviation of amperes consumed, and average and standard deviation of remaining voltage. Therefore, observe the notable amount of information delivered in each transmission. The Hash/Auth field was used for authentication and encryption purposes, hence the exchanged data were end-to-end encrypted.

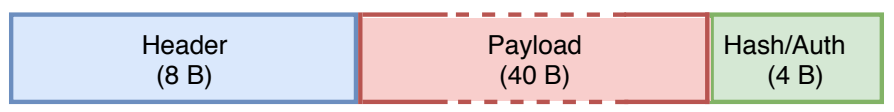

Figure 9. Transmitted frame structure.

As performance metrics, we have considered both the Received Signal Strength Indicator (RSSI) and the Packet Delivery Ratio (PDR). These metrics were studied under different LoRa's SF configurations, in order to analyze the most proper set-up for the scenario under evaluation (Figure 6). Concretely, we have made use of two values for the SF, namely, 7 and 12. SF7 permits greater data-rates at the expense of reducing the link robustness and vice versa. Considering the frame length of $52 \mathrm{~B}$ 
employed in our experiments (Figure 9), both the data-rate and time-on-air (ToA) of each transmission are shown in Table 1, which presents in bold the values corresponding to the evaluated configurations. Finally, we have also taken into consideration the power consumption of our on-board module in order to provide an estimation of its autonomy given that it is powered by batteries.

Table 1. LoRa (Long Range) transmissions' data-rate and ToA (Time-on-air) for the employed payload and BW (Bandwidth) of $125 \mathrm{kHz}$.

\begin{tabular}{cccc}
\hline SF (Spreading Factor)/DR (Data-Rate) & Employed Payload (B) & Data-Date (bps) & ToA (ms) \\
\hline $\mathbf{1 2 / 0}$ & $\mathbf{5 2}$ & $\mathbf{1 4 6 . 1}$ & $\mathbf{2 4 6 5 . 7}$ \\
$11 / 1$ & 52 & 261.4 & 1314.8 \\
$10 / 2$ & 52 & 584.2 & 616.4 \\
$9 / 3$ & 52 & 1359.2 & 328.7 \\
$8 / 4$ & 52 & 2738.1 & 184.8 \\
$\mathbf{7 / 5}$ & $\mathbf{5 2}$ & $\mathbf{4 8 4 4 . 7}$ & $\mathbf{1 0 2 . 6}$ \\
\hline
\end{tabular}

\section{Results}

In this section, the outcomes obtained in our validation tests are presented. As stated above, we have tracked a series of Optimist Class training sessions by placing a sensing and communication module on-board a number of sailboats (see Figure 5). Thus, firstly, a coverage study within the port area was made in order to analyze the impact of big buildings and other heavy machinery, e.g., cranes. Figures 10 and 11 present the RSSI level (received in the base station), obtained in different monitored training sessions with the two adopted LoRa configurations. Concretely, Figure 10 corresponds to the results attained with the following LoRa configuration: $\mathrm{SF}=12, \mathrm{CR}=4 / 5$, and $\mathrm{BW}=125 \mathrm{kHz}$, while Figure 11 shows the measured RSSI level corresponding to this configuration: $\mathrm{SF}=7, \mathrm{CR}=4 / 5$, and $\mathrm{BW}=125 \mathrm{kHz}$. Observe that both configurations permit covering the whole port area with good levels of coverage. However, a non-coverage zone was detected in both experiments (marked in red). This small area was surrounded by tall buildings that blocked the transmissions from the sailboats to the base station (small dots in the figures). Comparing both LoRa configurations, observe that, by using SF7, a greater data transmission frequency can be obtained (more sampling points in Figure 10 in comparison with Figure 11). This behavior is due to the data-rate attained with SF7 is greater than that of SF12, which permitted transmitting more tracking packets during the experiment. Regarding the robustness of the transmission links, Table 2 presents the PDR attained in both tests. In the studied area, we did not notice any packet loss with the exception of the non-coverage area mentioned above. Observe that we have considered both areas for calculating the PDR. The PDR level attained with SF7 is lower than that of SF12 because, due to the greater data-rate of SF7, in the non-coverage area more packets were sent (and not received) with this configuration. However, although not presented here, the PDR attained without considering the non-coverage area was almost $100 \%$ in both scenarios. 


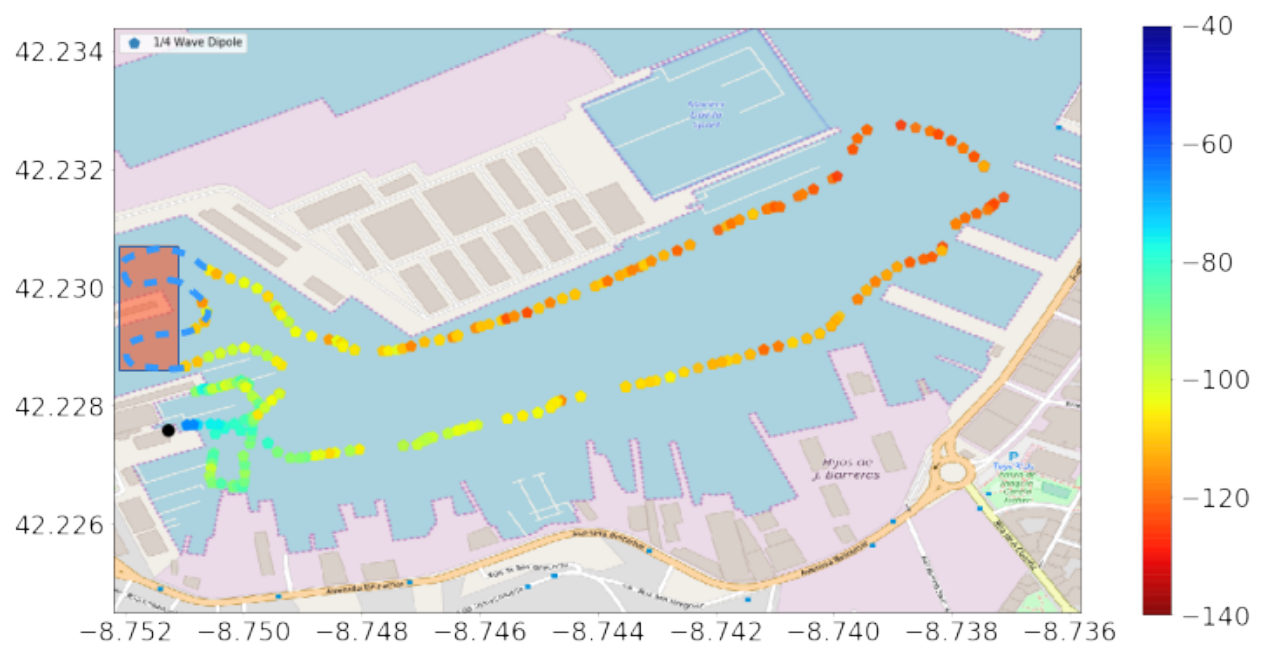

Figure 10. RSSI (Received Signal Strength Indicator) reception levels $(\mathrm{dBm})$ in the port area with LoRa's SF7.

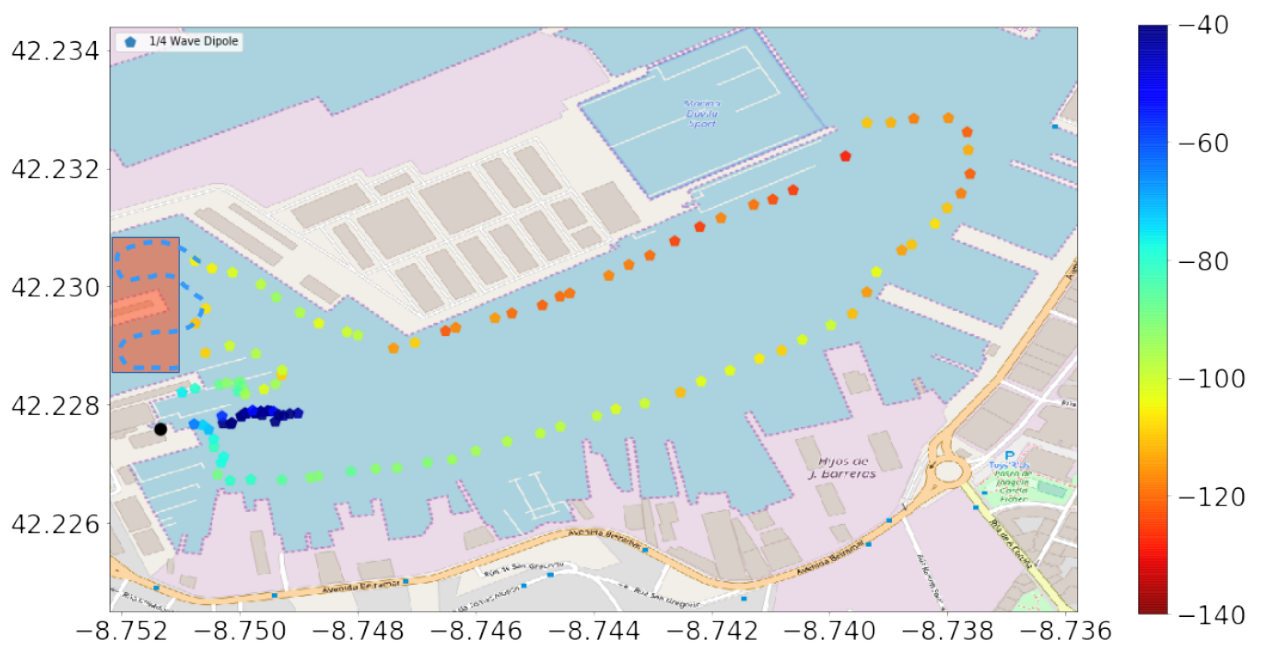

Figure 11. RSSI reception levels (dBm) in the port area with LoRa's SF12.

Table 2. Packet Delivery Ratio (PDR) in the port area.

\begin{tabular}{ccc}
\hline & SF7 & SF12 \\
\hline Packets Received & 2900 & 275 \\
Packets Transmitted & 4046 & 283 \\
PDR & $71.67 \%$ & $97.17 \%$
\end{tabular}

In order to validate the afore-discussed results, a regular Optimist training session was tracked. In this case, five sailboats (Figure 5) were simultaneously tracked during a period of $1 \mathrm{~h}$ in order to obtain their gathered data which were visualized by means of the monitoring dashboard (Figure 8). Thereby, Figure 12 presents the sampling points and the corresponding RSSI measured by the base station. Please note that, due to the good results attained in the previous experiment, a LoRa's SF7 was employed in this test and the sailboats sent their monitoring data every $5 \mathrm{~min}$. With this experiment, we aim to show the validity of the proposed infrastructure for tracking activities inside the port by means of the LoRa technology. During a real race, the sampling period would be shorter as that employed in the previous experiment.

Regarding the most proper LoRa configuration, as explained in previous sections, SF12 provides a superior robustness than SF7. For that reason, one could decide to always make use of the most reliable configuration $(\mathrm{SF}=12)$. However, there is an important trade-off that should be taken into account; 
the link robustness with this LoRa configuration is achieved at the expense of severely decreasing the transmission data-rate and, consequently, increasing the ToA of each transmitted message (see Table 1). This fact is not negligible in the case of having an important number of nodes simultaneously transmitting to the same base station due to potential interfering signals [29]). In addition, due to duty-cycle restrictions associated with the unlicensed transmission bands, employing low data-rates (high SF) implies the use of short payloads; hence, the information included in each transmitted frame is limited (Table 1). Therefore, when possible, it is preferred to employ low values for the SF (high data-rates) in order to increase the achieved throughput.

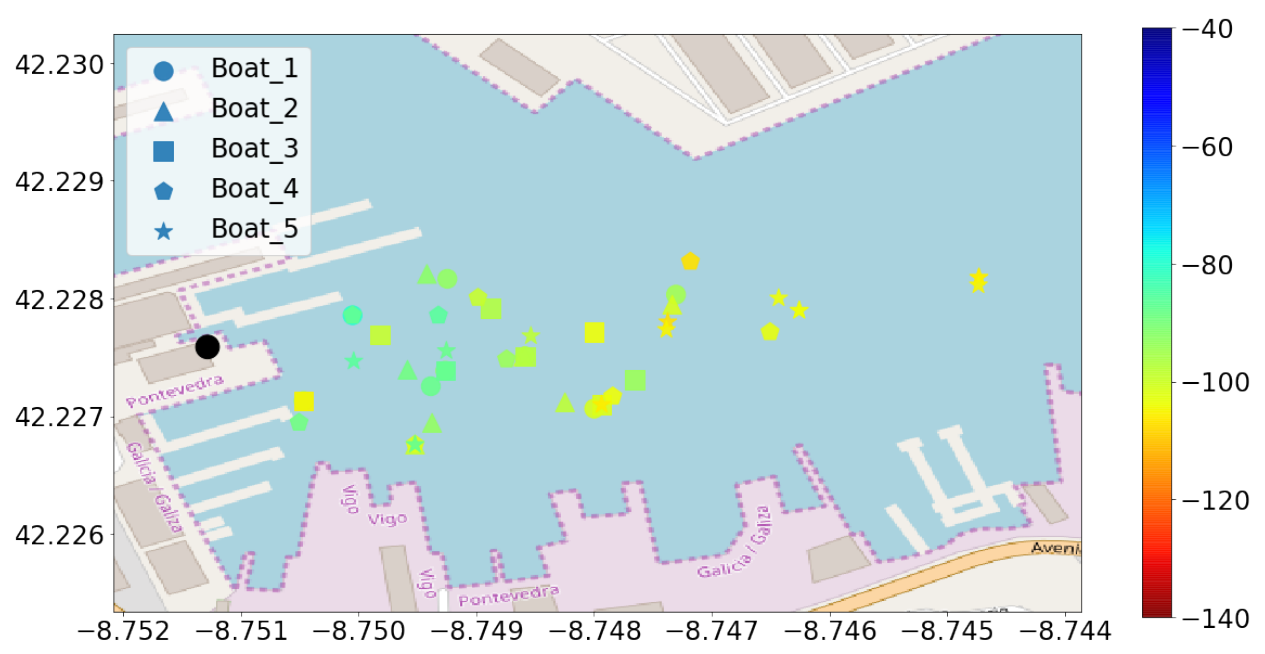

Figure 12. RSSI reception level $(\mathrm{dBm})$ attained during the tracking of an Optimist training session.

Another test was also conducted in order to evaluate the transmission range in open sea. In this case, we employed SF12 in order to evaluate the maximum distance reached by using the deployed architecture. The RSSI levels attained in this experiment are presented in Figure 13. Observe that, due to the limitations of the sailboats under study, they had to sail close to the shore for safety reasons. The maximum coverage range attained in this scenario was $4 \mathrm{~km}$. Although other works [10] have shown greater transmission distances in land, we justify the attained outcomes by the adverse propagation conditions around the base station, due to close big obstacles, as well as the proximity of the on-board transmission equipment to the water, as explained in the previous section. However, we consider that a range of $4 \mathrm{~km}$ from the port is long enough for monitoring maneuvers inside the port or activities in areas close to the shore, especially for boats with a stringent payload and energetic restrictions such as the sailboat under study (Optimist Class) or radio-control devices.

Finally, regarding the energy efficiency of this proposal, we have measured the power consumption of the end-device when transmitting packets every $5 \mathrm{~s}$ and employing LoRa's SF12. In these measurements, all the sensors were connected to the board and active. With this configuration, we attained a power consumption of $140 \mathrm{~mA}$, with a voltage of $5 \mathrm{~V}$. This consumption level permitted an autonomy of around $6.5 \mathrm{~h}$ with the employed power bank of $900 \mathrm{mAh}$, and would provide a battery lifetime of one day with an inexpensive power bank of 22,000 $\mathrm{mAh}$. This consumption level is far from other alternative communication technologies that may fit in the scenario under consideration such as the cellular ones $(2 \mathrm{G} / 3 \mathrm{G})$ as shown in $[30,31]$. 


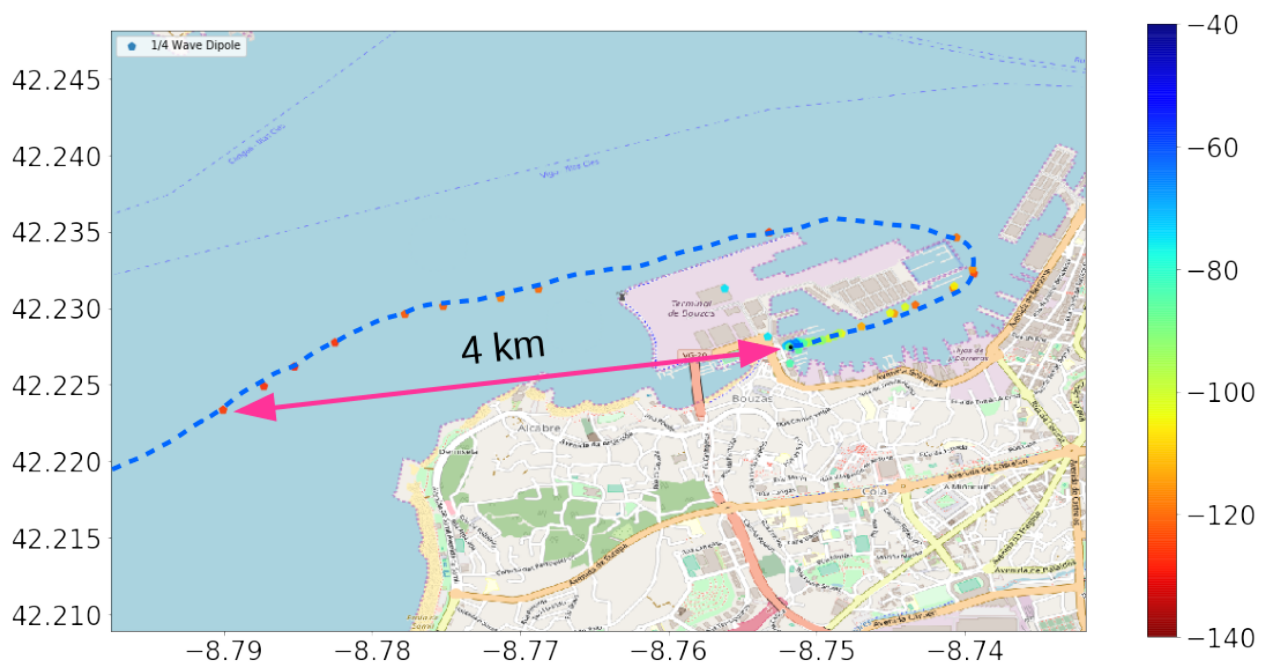

Figure 13. Coverage study in the open sea in terms of received RSSI (dBm).

\section{Conclusions}

Maritime communications may take advantage of the state-of-the-art communication technologies that are emerging under the umbrella of the IoT paradigm. For that reason, in this work, the use of LP-WAN solutions for providing ships with connectivity to land has been explored. The status of current existing solutions has been discussed and the advantages provided by these novel transmission technologies have been identified. Concretely, it has been found that the appropriateness of their use in boats with strong payload and energetic restrictions such as small sailboats or low-cost marine devices, e.g., radio-control boats.

Therefore, an LP-WAN-based architecture has been proposed and a field experiment has been conducted in order to validate this proposal. A sampling campaign has been carried out in order to evaluate the performance of LoRa, one of the most promising LP-WAN technologies, with the aim of monitoring the activities of Optimist Class sailboats. A real LoRa-based network has been deployed in the port of Vigo (Spain). The attained results showed the validity of LoRa aiming at tracking ships' maneuvers and monitoring a series of parameters within the port. Different LoRa configurations have been tested and discussed, concluding that, under good transmission conditions, high data-rates should be used in order to reduce the time-on-air of the transmissions. In addition, good levels of transmission reliability (above $97 \%$ of PDR) were attained with the most robust LoRa configuration.

A transmission range study has also been presented, showing a maximum distance of $4 \mathrm{~km}$ under the conditions of the deployed test-bench. We consider this distance long enough to monitor lightweight and small boats; hence, an online platform has been developed for the visualization of an Optimist Class race. Finally, the power consumption of end-devices has been evaluated, concluding that an autonomy of one day can be attained with low-cost batteries. As future work, we plan to extend this study in order evaluate the system performance in a different scenario, with bigger ships and, consequently, employing another piece of transmission equipment.

Author Contributions: R.S.-I. and A.F.S. designed the architecture and experiments. I.G.L. and C.S. led the implementation tasks. E.C. designed and deployed the cloud-based monitoring system. All the authors contributed to writing the paper.

Funding: This work has been supported by the European Union under the framework of the H2020 IoTCrawler project (contract 779852) and the Spanish Ministry of Economy and Competitiveness, through the PERSEIDES (ref. TIN2017-86885-R) project. In addition, it has been developed thanks to the collaboration of the LifeDEMOWAVE (LIFE14 CCM/ES/001209), E-CORYDORA (ITC-20151038) and MARRISK (0262_MARRISK_1_E) projects, the Asociación Tecnológica A Industriosa, and the Marine Instruments' Regata Solar.

Conflicts of Interest: The authors declare no conflict of interest. The founding sponsors had no role in the design of the study; in the collection, analyses or interpretation of data; in the writing of the manuscript; nor in the decision to publish the results. 


\section{Abbreviations}

The following abbreviations are used in this manuscript:

$\begin{array}{ll}\text { AUV } & \text { Autonomous Underwater Vehicles } \\ \text { BW } & \text { Bandwidth } \\ \text { CR } & \text { Coding Rate } \\ \text { CSS } & \text { Chirp Spread Spectrum } \\ \text { DTN } & \text { Delay Tolerant Network } \\ \text { GPS } & \text { Global Positioning System } \\ \text { GSM } & \text { Global System for Mobile communications } \\ \text { IoT } & \text { Internet of Things } \\ \text { ISM } & \text { Industrial, Scientific, and Medical } \\ \text { ITOW } & \text { Integer Time Of Week } \\ \text { kbps } & \text { kilobits per second } \\ \text { LDR } & \text { Light Dependent Resistor } \\ \text { LP-WAN } & \text { Low Power-Wide Area Network } \\ \text { LoRa } & \text { Long Range } \\ \text { MANET } & \text { Mobile Ad hoc Network } \\ \text { NANET } & \text { Nautical Ad hoc Network } \\ \text { PDR } & \text { Packet Delivery Ratio } \\ \text { RAN } & \text { Radio Access Network } \\ \text { RSSI } & \text { Received Signal Strength Indicator } \\ \text { SF } & \text { Spreading Factor } \\ \text { ToA } & \text { Time-on-Air } \\ \text { UAV } & \text { Unmanned Aerial Vehicles } \\ \text { UHF } & \text { Ultra High Frequency } \\ \text { USF } & \text { Unmanned Surface Vehicles } \\ \text { VHF } & \text { Very High Frequency } \\ & \end{array}$

\section{References}

1. Bekkadal, F. Future maritime communications technologies. In Proceedings of the OCEANS 2009-EUROPE, Bremen, Germany, 11-14 May 2009; pp. 1-6. [CrossRef]

2. Zolich, A.; Palma, D.; Kansanen, K.; Fjørtoft, K.; Sousa, J.; Johansson, K.H.; Jiang, Y.; Dong, H.; Johansen, T.A. Survey on communication and networks for autonomous marine systems. J. Intell. Robot. Syst. 2018, 1-25. [CrossRef]

3. Lees, G.; Williamson, W. Handbook for Marine Radio Communication, 6th ed.; Informa Law from Routledge; Taylor et Francis Group: Oxford, UK, 2015.

4. Guerra, A.G.; Francisco, F.; Villate, J.; Aguado Agelet, F.; Bertolami, O.; Rajan, K. On small satellites for oceanography: A survey. Acta Astronaut. 2016, 127, 404-423. [CrossRef]

5. Parker, J. European view of automated VHF/UHF radio systems-Marine spectrum usage alternatives and trends. IEEE Trans. Veh. Technol. 1977, 26, 223-226. [CrossRef]

6. $\mathrm{Xu}, \mathrm{Y}$. Quality of service provisions for maritime communications based on cellular networks. IEEE Access 2017, 5, 23881-23890. [CrossRef]

7. Sanchez-Iborra, R.; Cano, M.D. State of the art in LP-WAN solutions for industrial IoT services. Sensors 2016, 16, 708. [CrossRef] [PubMed]

8. Cao, B.; Zhao, J.; Yang, P.; Ge Lv, Z.; Liu, X.; Min, G. 3D Multi-objective deployment of an industrial wireless sensor network for maritime applications utilizing a distributed parallel algorithm. IEEE Trans. Ind. Inf. 2018. [CrossRef]

9. Geng, X.; Wang, Y.; Feng, H.; Zhang, L. Lanepost: lane-based optimal routing protocol for delay-tolerant maritime networks. China Commun. 2017, 14, 65-78. [CrossRef]

10. Sanchez-Iborra, R.; Sanchez-Gomez, J.; Ballesta-Viñas, J.; Cano, M.D.; Skarmeta, A. Performance evaluation of LoRa considering scenario conditions. Sensors 2018, 18, 772. [CrossRef] [PubMed]

11. LoRa-Alliance. Available online: https://www.lora-alliance.org/ (accessed on 19 December 2018). 
12. Petäjäjärvi, J.; Mikhaylov, K.; Pettissalo, M.; Janhunen, J.; Iinatti, J. Performance of a low-power wide-area network based on LoRa technology: Doppler robustness, scalability, and coverage. Int. J. Distrib. Sens. Netw. 2017, 13, 1-16. [CrossRef]

13. International Optimist Dinghy Association. Available online: http://www.optiworld.org/ (accessed on 21 December 2018)

14. Yang, X.; Liu, K.; Cui, Y.; Zhang, J. Modeling and simulation for maritime surveillance sensor networks. In Proceedings of the 2010 10th International Symposium on Communications and Information Technologies, Bradford, UK, 29 June-1 July 2010; pp. 215-219. [CrossRef]

15. Ahmadi, A.; Bigdeli, A.; Baktashmotlagh, M.; Lovell, B.C. A wireless mesh sensor network for hazard and safety monitoring at the Port of Brisbane. In Proceedings of the 37th Annual IEEE Conference on Local Computer Networks, Clearwater, FL, USA, 22-25 October 2012; pp. 180-183. [CrossRef]

16. O'Reilly, T.C.; Kieft, B.; Chaffey, M. Communications relay and autonomous tracking applications for Wave Glider. In Proceedings of the OCEANS 2015, Genova, Italy, 18-21 May 2015; pp. 1-6. [CrossRef]

17. Zainuddin, Z.; Wardi; Nantan, Y. Applying maritime wireless communication to support vessel monitoring. In Proceedings of the 4th International Conference on Information Technology, Computer, and Electrical Engineering (ICITACEE), Semarang, Indonesia, 18-19 October 2017; pp. 158-161. [CrossRef]

18. Kim, Y.; Kim, J.; Wang, Y.; Chang, K.; Park, J.; Lim, Y. Application scenarios of nautical ad hoc network for maritime communications. In Proceedings of the OCEANS 2009, Biloxi, MS, USA, 26-29 October 2009.

19. Mohsin, R.J.; Woods, J.; Shawkat, M.Q. Density and mobility impact on MANET routing protocols in a maritime environment. In Proceedings of the Science and Information Conference (SAI), London, UK, 28-30 July 2015; pp. 1046-1051. [CrossRef]

20. Lin, H.-M.; Ge, Y.; Pang, A.-C.; Pathmasuntharam, J.S. Performance study on delay tolerant networks in maritime communication environments. In Proceedings of the OCEANS2010, Sydney, Australia, 24-27 May 2010; pp. 1-6. [CrossRef]

21. Lambrinos, L.; Djouvas, C.; Chrysostomou, C. Applying delay tolerant networking routing algorithms in maritime communications. In Proceedings of the IEEE 14th International Symposium on "A World of Wireless, Mobile and Multimedia Networks" (WoWMoM), Madrid, Spain, 4-7 June 2013; pp. 1-6. [CrossRef]

22. Faria, M.; Pinto, J.; Py, F.; Fortuna, J.; Dias, H.; Martins, R.; Leira, F.; Johansen, T.A.; Sousa, J.; Rajan, K. Coordinating UAVs and AUVs for oceanographic field experiments: Challenges and lessons learned. In Proceedings of the IEEE International Conference on Robotics and Automation (ICRA), Hong Kong, China, 31 May-7 June 2014; pp. 6606-6611. [CrossRef]

23. Bešlić, M.; Perković, T.; Stančić, I.; Pavlov, G.; Čagalj, M. eMoorings: Distributed low power wide area system to control moorings. In Proceedings of the 2nd International Multidisciplinary Conference on Computer and Energy Science (SpliTech), Split, Croatia, 12-14 July 2017.

24. Li, L.; Ren, J.; Zhu, Q. On the application of LoRa LPWAN technology in Sailing Monitoring System. In Proceedings of the 13th Annual Conference on Wireless On-demand Network Systems and Services (WONS), Jackson, WY, USA, 21-24 February 2017; pp. 77-80. [CrossRef]

25. Jovalekic, N.; Drndarevic, V.; Pietrosemoli, E.; Zennaro, I. Experimental Study of LoRa Transmission over Seawater. Sensors 2018, 18, 2853. [CrossRef] [PubMed]

26. LoRa-Alliance. LoRaWAN Specification; Technical report; LoRa Alliance: Beaverton, OR, USA, 2015.

27. Di Serio, A.; Buckley, J.; Barton, J.; Newberry, R.; Rodencal, M.; Dunlop, G.; O’Flynn, B.; Di Serio, A.; Buckley, J.; Barton, J.; et al. Potential of Sub-GHz Wireless for Future IoT Wearables and Design of Compact $915 \mathrm{MHz}$ Antenna. Sensors 2018, 18, 22. [CrossRef] [PubMed]

28. Sanchez-Iborra, R.; Sanchez-Gomez, J.; Santa, J.; Fernandez, P.J.; Skarmeta, A. Integrating LP-WAN communications within the vehicular ecosystem. J. Internet Serv. Inf. Secur. 2017, 7, 45-56. [CrossRef]

29. Georgiou, O.; Raza, U. Low Power Wide Area Network Analysis: Can LoRa Scale? IEEE Wirel. Commun. Lett. 2017, 6, 162-165. [CrossRef] 
30. Gupta, P.K.; Saraswat, P.; Kumar, C.S.; Chakrabarti, S.; Rajakumar, R.V. Measurement of power consumption in multimedia mobiles for various network activities in $2 \mathrm{G} / 3 \mathrm{G}$ networks. In Proceedings of the IEEE International Conference on Advanced Networks and Telecommunciations Systems (ANTS), Bangalore, India, 16-19 December 2012; pp. 141-144. [CrossRef]

31. Becona, J.P.; Pereira, A.S.; Vazquez, C.; Arnaud, A. A battery powered RTU: GPRS vs 3G comparison. In Proceedings of the IEEE URUCON, Montevideo, Uruguay, 23-25 October 2017; pp. 1-4. [CrossRef]

(C) 2018 by the authors. Licensee MDPI, Basel, Switzerland. This article is an open access article distributed under the terms and conditions of the Creative Commons Attribution (CC BY) license (http:/ / creativecommons.org/licenses/by/4.0/). 